\title{
Saúde infantil: tendências e determinantes na cidade de São Paulo na segunda metade do século $X X$
}

\section{Children's health: trends and determinants in $\mathrm{S}$. Paulo city, Brazil, in the second half of the $20^{\text {th }}$ century}

“É por demais evidente o pouco que se sabe sobre as condições de saúde e de nutrição da população brasileira. ... pouco também se conhece sobre a heterogeneidade dos problemas de saúde dos vários segmentos da sociedade ... e menos ainda se sabe de eventuais mudanças ao longo do tempo. " 8 Com esse parágrafo, pouco animador, iniciávamos, há uma década, o relato dos resultados de pesquisa de campo realizada para “... obter um diagnóstico seguro das condições de saúde e de nutrição prevalecentes em meados da década de 80 entre as crianças residentes no Município de São Paulo”.

Nos últimos dez anos, o panorama das estatísticas brasileiras de saúde melhorou consideravelmente, em particular com relação a indicadores da saúde infantil. Tal transformação se deveu, sobretudo, à disseminação por todo o País de pesquisas de campo como a realizada em São Paulo em 1984/85. No plano nacional, tivemos a Pesquisa Nacional sobre Saúde Materno-infantil e Planejamento Familiar, de 1986, a Pesquisa Nacional sobre Saúde e Nutrição, de 1989, e a Pesquisa Nacional sobre Saúde e Demografia, de 1996. No plano regional, merecem destaque os inquéritos estaduais sobre saúde e nutrição na infância promovidos pelo Unicef no Nordeste do País e, por suas características inovadoras, os estudos prospectivos das coortes de recém-nascidos do Município de Pelotas, no Rio Grande do Sul. A realização dessas pesquisas e as análises empreendidas a partir das mesmas, ${ }^{1-4,6,9-18}$ além de apontarem expressivas melhorias em determinados indicadores, não mais autorizam afirmações como as que fazíamos no início dos anos 80; ao contrário, pode-se afirmar que a situação do País quanto à disponibilidade e à confiabilidade de indicadores de saúde na infância é, hoje, bastante superior àquela usualmente encontrada em países não desenvolvidos.

Todavia há limitações, e elas são importantes. De início, há de notar-se que os custos e as dificuldades operacionais do diagnóstico em campo têm levado, com frequiência, a investigar-se um conjunto restrito de indicadores que nem sempre abrange todo o espectro de problemas relevantes para a saúde infantil. Vê-se, também, que ainda são poucos os estudos seqüenciais realizados sobre amostras da mesma base populacional, estudos esses que permitiriam estabelecer, com confiança, a tendência temporal de diferentes indicadores de saúde. Ainda mais raros são os estudos sobre a evolução da equiidade na distribuição social dos indicadores e sobre os fatores e mecanismos que, em nosso meio, seriam responsáveis pela melhoria ou piora da saúde infantil. 
O esforço de investigação relatado nos artigos deste suplemento foi planejado de modo a preencher, ao menos, parte das lacunas identificadas acima. O componente principal desse esforço foi a repetição da pesquisa de campo realizada na cidade de São Paulo em meados da década de 80. Assim, de setembro de 1995 a setembro de 1996 (para que pudessem ser captadas variações sazonais nos indicadores de saúde) voltamos a estudar, em grande detalhe e com a mesma metodologia, uma amostra representativa das crianças de zero a cinco anos de idade residentes na cidade. Como no estudo anterior, submetemos a referida amostra de crianças a um cuidadoso e abrangente exame clínico, complementado por testes laboratoriais, capaz de identificar os principais problemas de saúde na infância. Ao exame clínico, seguiu-se a avaliação pormenorizada da alimentação infantil e de outros determinantes da saúde da criança, compreendendo antecedentes reprodutivos, condições ao nascer, características do ambiente domiciliar e peridomiciliar, saneamento do meio e disponibilidade e qualidade da assistência à saúde, entre outros. Um detalhado questionário socioeconômico permitiu a identificação da origem social das crianças (classes ou frações de classe social) e a descrição de atributos familiares que condicionam a saúde infantil, entre os quais a renda e o nível de escolaridade dos familiares da criança.

Visando ampliar o período de referência para o estudo da tendência secular de indicadores de saúde na infância, voltamos a utilizar estatísticas fornecidas por um estudo pioneiro sobre alimentação e nutrição na infância realizado na cidade de São Paulo em 1973/74. ${ }^{5}$ Com o mesmo propósito, recuperamos o banco de dados relativo à cidade de São Paulo do Estudo Nacional da Despesa Familiar - ENDEF, realizado em 1974/75. Resultados de um inquérito que realizamos em meados da década de 70 em uma amostra probabilística das maternidades então existentes na cidade de São Paulo, ${ }^{7}$ somados à série histórica das declarações de nascidos vivos do período 1993-1998 e a informações sobre condições de nascimento das crianças estudadas pelos inquéritos de 1984/85 e 1995/96, permitiram, dessa vez, um estudo minucioso da tendência secular do peso ao nascer na cidade.

Procurando estender o período de referência para o estudo da tendência secular de indicadores, incluímos, no projeto atual, o levantamento de fontes de dados que pudessem indicar, ainda que com menos detalhe e precisão, as condições da saúde e de nutrição na infância observadas na cidade nas décadas anteriores à de 70. Nesse sentido, recuperamos dados (idade, procedência e estatura) de uma amostra das fichas de alistamento militar das coortes masculinas de indivíduos nascidos na cidade entre 1950 e 1976.

No estudo relatado há dez anos, a partir da observação da evolução de alguns indicadores de saúde e da concomitante evolução da renda familiar e do acesso a serviços públicos de saúde e de saneamento, levantamos algumas hipóteses sobre possíveis determinantes da tendência secular, de modo geral favorável, da saúde infantil na cidade de São Paulo. Entretanto, tais hipóteses, estabelecidas com base em análises de plausibilidade, não puderam ser propriamente testadas em face do grau excessivo de agregação das informações anteriores à década de 80. Também não foi possível proceder a uma análise abrangente da evolução da equiidade na distribuição social dos indicadores, em vista da disponibilidade limitada de informações socioeconômicas anteriores à década de 80 .

No estudo atual fomos além quanto à análise da evolução da equiidade e quanto à investigação dos fatores e dos mecanismos responsáveis pela tendência secular da saúde infantil. Essencial foi dispormos, dessa vez, de duas pesquisas inteiramente comparáveis e planejadas para aferir tanto o estado de saúde e de nutrição das crianças da cidade quanto 
a situação em que se encontravam os fatores que, em potencial, determinam ou condicionam aquele estado.

A evolução da eqüidade na distribuição social dos indicadores foi determinada com base no comportamento de estratos sociais "equivalentes" nas pesquisas realizadas na cidade nas décadas de 70, 80 e 90. Três estratos sociais foram criados com esse propósito: aquele relativo ao terço das crianças mais pobres da cidade (o terço das crianças com menor renda familiar per capita em cada pesquisa), aquele relativo ao terço intermediário e aquele relativo ao terço das crianças mais ricas da cidade (o terço das crianças com maior renda familiar per capita em cada pesquisa).

Os procedimentos analíticos empregados para identificar a determinação da tendência secular dos diferentes indicadores de saúde são detalhados nos artigos que tratam da evolução particular de cada indicador. Em todos os casos, partimos de um modelo causal que articula, hierarquicamente, todos os fatores que potencialmente influenciam o estado de saúde retratado pelo indicador. A seguir, as principais hipóteses implícitas no modelo são testadas sobre a base de dados resultante do acoplamento das bases das pesquisas de 1984/85 e de 1995/96. Dessa avaliação, executada por meio de análises multivariadas de regressão, que levam em conta as especificidades do modelo hierárquico de determinação, ${ }^{19}$ resultam as variáveis efetivamente relevantes para a saúde infantil na cidade e no período. Dela também resultam estimativas do risco relativo de ocorrência das enfermidades (anemia, infestação por parasitas e doença respiratória, entre outras) segundo exposição a categorias dos determinantes (desmame precoce, ausência de saneamento e presença de fumantes no domicílio, por exemplo). O impacto de cada determinante sobre a evolução do indicador de saúde é, então, identificado calculando-se o aumento ou a redução na ocorrência da doença atribuível a variações temporais na freqüência do determinante. A redução ou o aumento na ocorrência da doença resulta da comparação entre os resultados obtidos ao se ponderar os riscos relativos da enfermidade segundo a distribuição da variável determinante na primeira e na segunda pesquisa. Assim, o impacto de um determinado fator sobre a tendência secular do indicador de saúde irá depender tanto da força de associação existente entre o fator e a doença (riscos relativos) quanto da intensidade da variação do fator ao longo do tempo.

Com os artigos preparados para este suplemento, espera-se que tenham sido reunidos os elementos necessários à compreensão da causalidade subjacente aos avanços (muitos) e retrocessos (poucos) que caracterizam a saúde das crianças da cidade de São Paulo na segunda metade do século XX.

\section{Carlos Augusto Monteiro Departamento de Nutrição da Faculdade de Saúde Pública da Universidade de São Paulo}

\section{REFERÊN CIAS}

1. Barros FC, Victora CG. Saúde materno-infantil em Pelotas, Rio Grande do Sul, Brasil: 1982-1993: uma década de transição. Cad Saúde Pública 1996;12 Supl 1:1-94.

2. BEM FAM, Brasil. Pesquisa N acional sobre Saúde Materno-Infantil e Planejamento Familiar - 1986. Rio de Janeiro: BEM FAM; 1987.

3. BEMFAM, Brasil. Pesquisa Nacional sobre Demografia e Saúde - 1996. Rio de Janeiro: BEM FAM/M acro International; 1997.
4. Benicio MHD'A, Cesar CLG, Gouveia NC. Perfil de morbidade e padrão de utilização de serviços de saúde das crianças brasileiras menores de cinco anos - 1989. In: Monteiro MFG, Cervini R, organizadores. Perfil estatístico de crianças e mães no Brasil. Aspectos de saúde e nutrição de crianças no Brasil - 1989. Rio de Janeiro: IBG E/U NICEF; 1992. p.79-96. 
5. lunes $M$, coordenador. Estado nutricional de crianças de 6 a 60 meses no Município de São Paulo. São Paulo: Instituto de Medicina Preventiva da Escola Paulista de Medicina/Instituto de Pesquisas Econômicas da USP; 1975.

6. Leão MM, Coitinho DC, Racine E, Costa LAL, Lacerda AJ. $O$ perfil do aleitamento materno no Brasil. In: Monteiro MFG, Cervini R, organizadores. Perfil estatístico de crianças e mães no Brasil. Aspectos de saúde e nutrição de crianças no Brasil - 1989. Rio de Janeiro: IBGE/U N ICEF; 1992. p.97-127.

7. Monteiro CA, Ishii M, Benicio MHD'A, Rea MF. A distribuição do peso ao nascer no Município de São Paulo. Rev Saúde Pública 1980;14:161-72.

8. Monteiro CA. Saúde e nutrição das crianças de São Paulo: diagnóstico, contrastes sociais e tendências. São Paulo: Hucitec/EDUSP; 1988.

9. Monteiro CA, Benicio MHD'A, Gouveia NC. Saúde e nutrição das crianças brasileiras no final da década de 80. In: Monteiro MFG, Cervini R, organizadores. Perfil estatístico de crianças e mães no Brasil: aspectos de saúde e nutrição de crianças no Brasil - 1989. Rio de Janeiro: IBGE/U NICEF; 1992. p.19-42.

10. Monteiro CA, Benicio MHD'A, lunes RF, Gouveia NC, Taddei JAAC, Cardoso MAA. O estado nutricional das crianças brasileiras: a trajetória de 1975 a 1989. In: Monteiro M FG, Cervini R, organizadores. Perfil estatístico de crianças e mães no Brasil. Aspectos de saúde e nutrição de crianças no Brasil - 1989. Rio de Janeiro: IBGE/U NICEF; 1992. p. 43-60.

11. Monteiro CA, Benicio MHD'A, Gouveia NC. Secular growth trends in Brazil over three decades. Ann Hum Biol 1994;21:381-90.

12. Monteiro CA, Mondini L, Souza ALM, Popkin BM. The nutrition transition in Brazil. Eur J Clin Nutr 1995;49:105-13.
13. Monteiro CA. Evolução da nutrição infantil nos anos 90 . In: Monteiro CA, organizador. Velhos e novos males da saúde no Brasil: a evolução do País e de suas doenças. $2^{\text {a }}$ ed. aum. São Paulo: HU CITEC/ NU PEN S-USP; 2000. p. 375-92.

14. M onteiro CA, Benicio MHD'A, Freitas IC. Evolução da mortalidade infantil e do retardo de crescimento nos anos 90: causas e impacto sobre desigualdades regionais. In: Monteiro CA, org. Velhos e novos males da saúde no Brasil: a evolução do país e de suas doenças. $2^{\text {a }}$ ed. aum. São Paulo: HU CITEC/NU PEN SUSP; 2000. p. 393-420.

15. M onteiro CA, M ondini L. Changing nature of nutritional disorders in the developing countries: the case of Brazil. In: Fitzpatrick DW, Anderson JE, L'Abbé ML, editors. From nutritional science to nutrition practice for better global health. Proceddings of the $16^{\text {th }}$ International Congress of Nutrition; 1998; Ottawa: Canadian Federation of Biological Societies; 1998. p. 263-5.

16. Monteiro CA, Conde W L. Time trends in overweight prevalence in children, adolescents and adults from less and more developed regions of Brazil. In: Ailhaud G Guy-Grand B, editors. Progress in obesity research: $8^{\text {th }}$ International Congress on O besity; 1999; London: John Libby; 1999. p. 665-71.

17. Simões CCS. 0 estudo dos diferenciais na mortalidade infantil segundo algumas características sócioeconômicas. In: Monteiro MFG, Cervini R, organizadores. Perfil estatístico de crianças e mães no Brasil. Aspectos de saúde e nutrição de crianças no Brasil 1989. Rio de Janeiro: IBGE/U NICEF; 1992. p. 61-78.

18. Victora CG, Barros FC, Vaughan JP. Epidemiologia da desigualdade. São Paulo: Hucitec; 1988.

19. Victora CG, Huttly SR, Fuchs SC, O linto MTA. The role of conceptual frameworks in epidemiological analysis: a hierarchical approach. Int J Epidemiol 1997;26:224-7. 